\title{
CIBERPLANIFICACIÓN, PROPIEDAD PRIVADA Y CÁLCULO ECONÓMICO*
}

Victor I. Espinosa

* DOI: https://doi.org/10.18601/01245996.v23n45.08. Recepción: 03-032021, modificación final: 06-05-2021, aceptación: 02-06-2021. Sugerencia de citación: Espinosa, V. I. (2021). Ciberplanificación, propiedad privada y cálculo económico, Revista de Economía Institucional, 23(45), 163-183.

a Doctor en Economía. Profesor, Universidad Autónoma de Chile, Santiago de Chile, [victor.espinosa@uautonoma.cl], [https://orcid.org/0000-00022481-5082]. 


\section{Ciberplanificación, propiedad privada y cálculo económico}

Resumen Este artículo revisa y discute las observaciones del profesor Maxi Nieto sobre algunos temas importantes de la moderna teoría del socialismo. En particular, su interpretación del argumento contra la "posibilidad" del cálculo económico sin derechos de propiedad privada desarrollado por la nueva generación de autores de la escuela austriaca. Centra la atención en cuestiones metodológicas para resaltar las deficiencias teóricas de algunas de las principales ideas defendidas por Nieto. También analiza su propuesta del ciber-comunismo como alternativa para superar el desafío del cálculo económico a través de las nuevas tecnologías de la información y la comunicación. Argumenta que su análisis es superficial y que el desafío de los austriacos sobre la imposibilidad del socialismo sigue siendo sólido.

Palabras clave: cálculo económico, mercado, planificación, socialismo; JEL: B53, P21

\section{Cyber planning, private property and economic calculation}

Abstract This article reviews and discusses Professor Maxi Nieto's observations on some major topics in in the modern theory of socialism. Notably, his interpretation of the argument against the "possibility" of economic calculus without private property rights developed by the new generation of Austrian school authors. It focuses on methodological issues to highlight the theoretical deficiencies of some of the leading ideas defended by Nieto. It also analyzes his proposal of cyber-communism as an alternative to overcome the challenge of economic calculation through the new information and communication technologies. It argues that his analysis is superficial and that the Austrian' challenge on the impossibility of socialism remains sound.

Keywords: economic calculation, markets, planning, socialism; JEL: B53, P21

\section{Ciber planeamento, propriedade privada e cálculo económico}

Resumo Este artigo revisa e discute as observações do professor Maxi Nieto sobre algumas questões importantes na teoria do socialismo moderno. Notavelmente, sua interpretação do argumento contra a "possibilidade" de cálculo econômico sem direitos de propriedade privada desenvolvido pela nova geração de autores da escola austríaca. Concentra em questões metodológicas para destacar as lacunas teóricas de algumas das principais ideias defendidas por Nieto. Analisa também sua proposta de ciber-comunismo como alternativa para superar o desafio do cálculo econômico por meio das novas tecnologias de informação e comunicação. Argumenta que sua análise é superficial e que o desafio austríaco sobre a impossibilidade do socialismo permanece sólido.

Palavras-chave: cálculo econômico, mercado, planeamento, socialismo;JEL: B53, P21 
T os teóricos de la planificación central consideran que el desafío Lde los economistas austriacos perjudica su posición. El análisis y el trabajo en los que se basan sus opiniones son quizá bien conocidos (Boettke, 2000). Hace poco esta revista publicó un escrito del profesor Maxi Nieto que expone un enfoque distinto de las tesis austriacas sobre la imposibilidad del socialismo “¿Es imposible el cálculo económico en el socialismo?” (Nieto, 2020). Este artículo resume y evalúa sus principales argumentos, y sostiene que su análisis es superficial y que el desafío austriaco sigue siendo sólido.

La primera parte resume las opiniones del profesor Nieto -que en gran medida son un asalto a lo que para él es el desafío austriaco-, quien reafirma la idea marxista del cálculo económico en tiempo de trabajo y especula sobre la "posibilidad" del ciber-comunismo: la planificación cibernética sin derechos de propiedad privada a través de las actuales tecnologías de la información y la comunicación (TIC) (Cockshott, 2017). Desde una metodología basada en la concepción dialéctica y materialista de la realidad, intenta dar respuesta a las propuestas de los austriacos que encuentra tan objetables.

La segunda parte evalúa sus argumentos. Esto no significa que en cada tema intentemos decidir si él o los autores que critica tienen razón, pues a menudo sus explicaciones no son concluyentes porque su objetivo es criticar algunos argumentos y no las políticas. Por tanto, en la evaluación primero se explica el fundamento metodológico del marxismo para revelar sus errores teóricos, y después se evalúan las propuestas del profesor Nieto en respuesta a la visión austriaca.

La discusión sobre la imposibilidad del ciber-comunismo sigue líneas similares, quizá la de principal interés o preocupación para algunos lectores, y se basa en las conclusiones de la evaluación. A veces el análisis es más detallado porque el autor parece no comprender qué es el "mercado" para los austriacos y malinterpreta la teoría de Huerta de Soto (2015) -obra que Nieto utiliza como referencia para su realizar su crítica a los austriacos- sobre el error intelectual de toda variante socialista y marxista.

\section{SÍNTESIS DEL ARTÍCULO}

La primera parte del artículo mencionado explora "el debate formal sobre el cálculo económico y la asignación racional de recursos en el socialismo" (Nieto, 2020, p. 129). El autor sostiene que en este debate se observan tres etapas. En la primera, Mises $(1920,1922,1949)$ sugirió que el cálculo económico en el socialismo es imposible, es decir, la 
planificación central no es posible, porque sin propiedad privada de los medios de producción, no habrá mercado de medios de producción; sin un mercado de medios de producción, estos no tendrán precios monetarios; $y$ sin precios monetarios que reflejen la escasez relativa de bienes de capital, los encargados de tomar las decisiones económicas serán incapaces de calcular sus usos alternativos. De modo que sin cálculo económico racional "el socialismo es irrealizable"(Mises, 1922, p. 135). Así, según Nieto, el desafío de Mises implica que es

imposible calcular los costos (reducir los insumos utilizados a un denominador común mediante alguna unidad de cuenta) y, por tanto, asignar de manera eficiente los recursos a los usos deseados, eligiendo entre las innumerables combinaciones posibles de factores de producción aquellas que minimicen los costos (Nieto, 2020, p. 129).

Para responder al desafío de Mises, los socialistas de mercado, como Lange, Lerner, Taylor, Dickinson, y Durbin, buscaron sustitutos del dinero a través del método de prueba y error o precios paramétricos (Lavoie, 1985; Caldwell, 1997). Pero sus opiniones se basaron en los modelos de equilibrio walrasiano y eficiencia paretiana, es decir, modelos estáticos en los que la información de la sociedad está dada y los resultados están contenidos en sus fórmulas. En otras palabras, en estos modelos no hay acción humana en el verdadero sentido de la palabra. Por eso, Nieto afirma que los teóricos socialistas hallaron erróneamente "una similitud formal entre las economías capitalista y socialista” (2020, p. 130). A este respecto, Arnaert (2018) señala que los socialistas de mercado no entendieron que el desafío de Mises no tiene relación con el paradigma neoclásico-keynesiano, debido a la esencia estática y poco realista de los supuestos matemáticos y econométricos. De hecho, nunca hubo realmente un debate (Huerta de Soto, 2004a).

La segunda etapa del debate que observa Nieto corresponde a la crítica de Hayek $(1935,1944,1945,1988)$ a los socialistas de mercado, quien "trasladó [...] el problema original de calcular costos (con alguna unidad de cuenta) para economizar recursos a otro [...] de coordinación social eficiente en un marco dinámico, cuya clave sería el descubrimiento, la transmisión y el uso de la información y el conocimiento disperso entre los agentes" (Nieto, 2020, p. 131). Para Hayek, la coordinación de los planes individuales de acción solo cobra sentido cuando el conocimiento de millones de seres humanos se transmite a través del sistema de precios. Sin embargo, el socialismo supone que una autoridad central única puede obtener y coordinar todo el conocimiento disperso en la sociedad. De modo que no es 
correcto suponer que una autoridad central puede disponer de todo este conocimiento. Los planificadores centrales pasan por alto que la dinámica de la sociedad moderna (fenómenos complejos) supera la capacidad de toda mente individual. Los modelos de planificación central consideran la creación y transmisión del conocimiento en sus ecuaciones como si fuese algo "dado" al alcance de los miembros del órgano de gobierno ${ }^{1}$.

Seguidamente, el profesor Nieto sugiere que "nunca se dio una respuesta eficaz y bien elaborada al planteamiento austriaco" (2020, p. 132). Sin embargo, las contribuciones de Mises y Hayek quedaron relegadas debido a "la hegemonía del enfoque neoclásico-keynesiano -el marco utilizado por los socialistas de mercado-y al aparente desarrollo económico de la Unión Soviética”.

El derrumbe de la órbita soviética en los años ochenta y noventa y un entorno político e ideológico más propicio a la libertad económica, llevaron a la tercera etapa del debate. Nieto sugiere que la posición de Jesús Huerta de Soto en su obra titulada Socialismo, Calculo Económico y Función Empresarial (2015) es la más completa de la nueva lectura austriaca. Para Huerta de Soto, "el problema económico fundamental es un problema empresarial de interacción humana, cuya clave es descubrir y movilizar la información necesaria, sobre nuevos fines y sobre los medios requeridos para alcanzarlos, clave que depende del libre ejercicio de la función empresarial" (Nieto, 2020, p. 133). En este sentido, Huerta de Soto afirma que:

el socialismo es un error intelectual, pues no cabe concebir que el órgano director encargado de intervenir mediante mandatos pueda hacerse con la información que es necesaria para coordinar la sociedad, y ello por los siguientes motivos: primero, por razones de volumen (es imposible que el órgano de intervención asimile conscientemente el enorme volumen de información práctica diseminada en las mentes de los seres humanos); segundo, dado el carácter esencialmente intransferible al órgano central de la información que se necesita (por su naturaleza tácita no articulable); tercero, porque, además, no puede trasmitirse la información que aún no se haya descubierto o creado por los actores y que solo surge como resultado del libre proceso de ejercicio de la función empresarial; y cuarto, porque el ejercicio de la coacción impide que el proceso empresarial descubra y cree la información necesaria para coordinar la sociedad (Huerta de Soto, 2015, pp, 99-100; citado por Nieto, p. 133).

${ }^{1}$ Algunos autores consideran que, si bien Hayek al inicio compartió el radicalismo de von Mises sobre la imposibilidad del cálculo económico en el socialismo, luego adoptó una posición más moderada. Sin embargo, Caldwell (2008) muestra que Hayek siempre sostuvo que el socialismo es "técnicamente" imposible. 
La segunda parte del artículo de Nieto critica los argumentos austriacos -sintetizados en Huerta de Soto- al ciber-comunismo como solución a la imposibilidad del cálculo económico en el socialismo. Su primera crítica a lo que él entiende como visión austriaca es que el problema del cálculo económico es consecuencia del individualismo metodológico, que solo tiene sentido en un sistema capitalista. Nieto dice que para la escuela austriaca "la esencia del proceso social" es la función empresarial y la búsqueda de oportunidades de ganancia, "un comportamiento que está lejos de un rasgo de la naturaleza humana", y es una "exigencia que impone un sistema mercantil" (ibíd., p. 137). Así, la función empresarial solo sería necesaria para resolver los problemas de cálculo económico y coordinación social creados por el capitalismo. De modo que el problema del cálculo económico no existiría en una comunidad socialista. Desde la perspectiva marxista, la esencia del proceso social no es la acción individual, pues es resultado de "reglas sociales" que no eligen los individuos.

La segunda crítica de Nieto es la existencia de un razonamiento circular, es decir, que los austriacos toman como premisa (la función empresarial) lo que se debería explicar (cómo y por qué se ejerce la función empresarial). Si los austriacos declaran "que la libre iniciativa de los individuos en el mercado (es decir, el ejercicio de la función empresarial) es fundamental para el funcionamiento del mercado", entonces el mercado es clave "para calcular los costos y resolver los problemas de información y coordinación que genera el propio mercado". Si los austriacos identifican la "sociedad" con el "mercado", "solo se puede concluir que "el cálculo racional es imposible". Pero es un error demostrar que el cálculo racional requiere el mercado [...] partiendo de la premisa de que el cálculo económico se basa en el mercado"(ibíd., pp. 137-138). Por ello, según Nieto, el individualismo metodológico y el razonamiento circular llevan a que la objeción de Mises a la posibilidad del cálculo económico en el socialismo solo se refiera a peculiaridades del capitalismo, que nada prueban sobre imposibilidad del socialismo.

Por último, Nieto argumenta que la tesis austriaca sobre la imposibilidad del socialismo se refiere a problemas superados por el desarrollo tecnológico. Propone el ciber-comunismo como sistema de planificación social sin derechos de propiedad privada a través de las nuevas tecnologías de la información y la comunicación (TIC) (big data, inteligencia artificial, internet de las cosas, supercomputadores). "La información se puede movilizar de manera más flexible y rápida en una economía cibernéticamente planificada como la que permite 
la tecnología actual", es decir, "este tipo de información no es un problema para el socialismo” (ibíd., p. 140). Así, el cálculo económico sería posible en términos de tiempo de trabajo. De modo que la crítica austriaca a los modelos planométricos de optimización matemática sería válida "para los modelos de socialismo de mercado de matriz neoclásica, pero no para una economía socialista cibernéticamente planificada sin procesos de mercado" (ibíd., p. 147).

El ciber-comunismo no solo es compatible con el criterio austriaco de eficiencia dinámica - la coordinación de los planes individuales de acción- sino que proporciona un marco institucional más adecuado para impulsar el desarrollo genuino de las preferencias y necesidades reales de la población. En particular, la producción ciber-comunista incluiría en tiempo real los cambios en la demanda final de bienes de consumo para coordinar las disposiciones sociales en toda la cadena de ciber-industrias. Esto invalidaría la objeción de Hayek, porque los desajustes serían resueltos por las tecnologías y "tampoco habría ninguna traba que impida canalizar la iniciativa creadora de los individuos de manera descentralizada” (ibíd., p. 148).

En el ciber-comunismo, "cada cual determina sus preferencias de consumo y su plan de vida, mientras que las decisiones que afectan al colectivo se toman democráticamente, no por imposición de una minoría propietaria" (ibíd.). En este sistema, continúa Nieto, puede haber "una pluralidad de órganos para organizar la producción, y por ensayo y error pueden corregir los posibles desajustes, quizá mucho menores debido a que no se tomarían decisiones paralelas ni existiría incertidumbre empresarial" (ibíd.). Y acaba diciendo que "trabajar bajo órdenes en un mando de propiedad social sería tan coactivo como trabajar en una empresa privada... pero, como hemos advertido, esa información se puede captar perfectamente en un marco de propiedad social mediante las nuevas tecnologías" (ibíd., p. 149).

\section{UNA EVALUACIÓN}

Pasamos ahora al análisis crítico de las opiniones del profesor Nieto que hemos resumido. En cada tema se sigue el patrón esbozado al comienzo: se describen sus errores; se hace una comparación de su propuesta teórica y, a veces se hacen algunos comentarios de los argumentos de los autores que critica.

Pero primero se abordan algunos problemas metodológicos del profesor Nieto que están presentes en todo el artículo. Este análisis preliminar favorecerá la comprensión acerca de los errores teóricos 
en su crítica a los austríacos. En particular, recurre a la concepción dialéctica y materialista de la realidad de Karl Marx, basada en Hegel, que hace contrastes arbitrarios de la realidad y lleva inexorablemente al determinismo materialista: una visión estática, donde el futuro está implícito en el presente. Dado que la dialéctica significa tendencias conflictivas o contradictorias, una al lado de la otra e incluso en el mismo conjunto de situaciones, cualquier predicción se puede justificar ${ }^{2}$. No hay forma de falsearla porque se refiere a un momento no especificado de un supuesto futuro radicalmente alterado. En otras palabras, es un método "que permite considerar el vaivén de un péndulo como la imagen de un gran principio filosófico" (Gray, 1946, p. 300). En este sentido, Marx escribió a Engels: "es posible que me desacrediten. Pero en ese caso, todavía será posible salir adelante con la ayuda de un poco de dialéctica. No hace falta decir que redacté mis pronósticos de tal manera que demostraría tener razón también en el caso contrario" (Shafarevich, 1980, p. 210).

No hay lugar donde Nieto esté más confundido o vacilante que en su base: el concepto de "materialismo histórico". El determinismo de la historia se relaciona con las "fuerzas productivas materiales" como motor unicausal de los acontecimientos y cambios históricos, tales como las relaciones sociales de producción y el resto de las formas de conciencia, con independencia de las voluntades de las personas. La tecnología determina la superestructura económica de la sociedad. Así, la única manera de que haya cambio social es a través del cambio tecnológico. Pero este argumento es falaz desde el principio, pues no se pregunta ¿de dónde viene la tecnología?, ¿cómo cambian las tecnologías?, ¿quién las pone en práctica? Nieto no responde estas preguntas. Si atribuyera el estado de la tecnología o el cambio tecnológico a la acción individual se desmoronaría todo su sistema, pues la conciencia individual es la fuerza que impulsa la producción material y no al revés ${ }^{3}$.

2 Marx razonó de la tesis a la negación de la tesis y a la negación de la negación. La tesis, al comienzo, es la propiedad de los medios de producción por cada trabajador. Este era el estado de cosas en una sociedad en la que el trabajador es un agricultor independiente o un artesano que posee sus herramientas de trabajo. La negación de la tesis -de la propiedad privada- es la situación en que las herramientas ya no son propiedad de los trabajadores sino de los capitalistas. La negación de la negación es la propiedad de los medios de producción por toda la sociedad. Razonando así, Marx pensó que había descubierto "la ley de la evolución histórica” a través del "socialismo científico" (Marx, 1843; Marx y Engels, 1932; Mises, 2006).

${ }^{3}$ A partir del determinismo tecnológico, Marx dijo que: "el molino de mano resulta en la sociedad feudal; el molino de vapor, el capitalismo industrial" 
A partir de este enfoque metodológico, Nieto construyó sus críticas a las propuestas de los austriacos basado en tres errores teóricos de los economistas clásicos que Marx $(1867,1885,1894)$ llevó hasta sus últimas consecuencias lógicas (Cachanosky, 1994, 1995; Rothbard, 1995; Steele, 2013). Primero, la teoría del valor trabajo mezclada con la tesis objetivista del costo de producción llevó a Nieto a sugerir que el valor de las cosas surge del trabajo contenido en ellas. ¿Es el valor objetivo? La teoría moderna del valor indica que lo que los seres humanos consideran valioso es subjetivo, es decir, valoran los bienes dependiendo de cómo satisfacen sus preferencias subjetivas según sus planes individuales de acción ${ }^{4}$.

¿Son los costos objetivos? E1 costo solo surge de las decisiones individuales. Los costos son subjetivos porque anticipan ex ante oportunidades relegadas por la decisión adoptada: el costo de oportunidad. Si el actor "piensa" que el valor de su fin alcanzado superó el valor de los fines sacrificados (costos), el actor ha conseguido una ganancia subjetiva. En caso opuesto, el actor cometió un error y obtuvo una pérdida subjetiva. Al menos de forma transitoria, las ganancias indican al actor que sus elecciones de fines y medios ha sido correctas (incentivo para actuar), y las pérdidas indican que ha desperdiciado recursos (incentivos para detener la acción). Este análisis subjetivo de pérdidas y ganancias guía a las personas hacia el logro de fines cada vez más valiosos.

Segundo, la metáfora del valor trabajo permitió que Nieto acusara al capitalismo de producir por las ganancias -bienes con valor de cambio- y no para el uso -bienes con valor de uso-. Antes de 1870, cuando Jevons, Menger, y Walras expusieron la teoría de la utilidad

(1847, p. 151). Además de los defectos lógicos, la doctrina materialista es absurda. Obviamente, el molino de mano, "que gobernó en la antigua Sumeria, no 'dio' una sociedad feudal: hubo relaciones capitalistas mucho antes del molino de vapor. El determinismo tecnológico llevó a Marx a aclamar cada invención importante como la mágica "fuerza productiva material" que irremediablemente provocaría la revolución socialista” (Rothbard 1995, p. 374). Entonces, ¿cómo es posible explicar la existencia de la sociedad rastreando su origen en las fuerzas productivas materiales que por sí mismas solo pueden aparecer en el marco de un nexo social previamente existente? Marx no resolvió este problema en sus escritos. Ver Mises (1957, pp. 105-111).

${ }^{4}$ La diferencia fundamental entre la acción humana y los entes de la naturaleza es la categoría de finalidad. Siguiendo la teoría subjetiva del valor de Menger (1871): para que un bien sea útil debe existir una necesidad humana, un objeto que la satisfaga y el conocimiento subjetivo de que ese objeto existe o podría existir. El mismo bien físico puede ser útil en un momento e inútil en otro. Así, la utilidad y la escasez son elementos subjetivos en función de los planes de acción. 
marginal, muchos teóricos creían en la errada teoría del valor trabajo. Hoy, los economistas reconocen que una hora de trabajo de una persona promedio no crea el mismo valor que una hora de trabajo de Elon Musk. Algunas personas son más perspicaces y el trabajo no es homogéneo. Aunque todos tuvieran la misma productividad, la teoría del valor trabajo seguiría siendo falaz. El valor de los bienes está determinado por la percepción subjetiva individual de su utilidad marginal. Desde la perspectiva de la acción humana, los bienes no poseen un valor intrínseco. Por eso, la división entre valor de cambio y valor de uso es un paradigma teórico obsoleto que se suele utilizar para imponer una escala valorativa a los demás.

Tercero, la segmentación de la sociedad en clases, la división de los procesos de producción y distribución en una economía de suma cero, fueron los ingredientes de Nieto para justificar la teoría de la lucha de clases. El pensamiento de cada individuo, sus valores y teorías, están determinados por su clase. Pero estos postulados son paradójicos. Si la sociedad está dividida en clases antagónicas, ¿qué sucede con el propio sistema marxista? Es una ironía que Marx, Engels y todos los marxistas destacados sean burgueses. ¿Por qué se han de aceptar algunas de sus ideas como verdaderas? Si la estructura lógica de la mente varía según la clase social o la raza, ¿cuál es la definición científica de clase? ¿Cómo cambia la estructura mental? ¿Por qué hay personas con mentalidad distinta a la de su clase? ¿Cuál es el límite de cada una? El polilogismo es un blindaje contra las críticas, puesto que no cabe concebir estructuras lógicas diferentes. Las teorías solo se pueden refutar demostrando sus errores; es irrelevante quién sea su autor. Además, la ley de asociación de Ricardo -que los individuos se benefician si se especializan en lo que tienen una ventaja comparativa relativa mayor e intercambian- y una comprensión básica de la teoría económica bastan para demostrar que los vínculos contractuales no son juegos de suma cero (Mises, 1949, pp. 158-160).

En resumen, la metodología marxista de Nieto es un entramado de errores y conexiones endebles (ver, p. ej., Böhm-Bawerk, 1896; Mises, 1949; Reisman, 2018). Los enfoques de la dialéctica, el determinismo tecnológico y la teoría del valor trabajo son demasiado extravagantes para defenderlas con seriedad. La reivindicación de estas doctrinas es la actitud del fiel religioso y no la del científico social.

\section{SOBRE EL RAZONAMIENTO CIRCULAR}

Una vez examinados los problemas metodológicos de Nieto, ahora se debe demostrar los errores en su crítica a los austríacos. Su primera 
crítica concierne a la concepción del individualismo metodológico que "pretende explicar las propiedades del medio social como resultado de las acciones individuales [...] Pero como advertimos, la acción humana depende del marco institucional en el cual se desarrolla. Por eso, para Marx, la esencia del proceso social no puede ser nunca la mera acción individual" (Nieto, 2020, p. 138).

En la sección anterior explicamos lo paradójico de la perspectiva dialéctica y materialista de la historia, en la cual los individuos son máquinas sin voluntad. Si los marxistas tienen razón y solo existe el colectivo dinámico sobre el individuo estático, ¿por qué argumentan como individuos (o grupo de individuos)? Esta tesis no resiste el análisis lógico. Por eso, los conceptos holistas como “sociedad" o "Estado" no tienen existencia ontológica (Evans, 2010). Si solo los individuos eligen, la forma de entender los fenómenos sociales -su estructura y su cambio- y conceptos culturales como el de "sociedad" es analizar la acción individual. Si perdemos de vista a los individuos, la "sociedad" no tiene sentido. Entonces, la esencia de los fenómenos sociales, lo que mueve a la sociedad, son las acciones de los individuos cuando actúan. El concepto económico de acción humana "designa la conducta de los seres humanos como un proceso continuo que es ideado por el actor de antemano, es decir, que se basa en un proyecto preconcebidos subjetivamente" (Schutz 2011, p. 76).

$\mathrm{E} 1$ profesor Nieto añade que "el principal efecto del individualismo metodológico es la naturalización del medio social capitalista, al que considera eterno y, paradójicamente, también como culminación de la historia del desarrollo humano" (Nieto, 2020, p. 138.). Otro autor podría replicar que el holismo metodológico es la naturalización del comunismo, al que considera eterno y, paradójicamente, como la culminación de la historia del desarrollo humano. ¿ Cuál es la relevancia científica de estas opiniones? Ninguna. En el mejor de los casos tienen utilidad ideológica.

A diferencia del materialismo de la teoría marxista, la economía es una ciencia libre de juicios de valor, es decir, no disputa los fines de los individuos. Se limita a examinar los cursos de acción y sus efectos en la actividad económica a partir de "cadenas verbales de la lógica" (Rothbard, 1951; Selgin, 1988; Hoppe, 1995; Huerta de Soto 2004b; Espinosa, 2017). La ciencia económica estudia la actividad humana. Es la teoría general de las categorías de la acción en "cualquier" entorno institucional en el que se actúe. Confundir economía con apología del capitalismo es desconocer su objeto. 
Ahora, nos ocuparemos de la segunda crítica del profesor Nieto a los austríacos: la existencia de un "razonamiento circular" en la teoría austríaca moderna sintetizada en Huerta de Soto (2015). Sin embargo, esta afirmación es incorrecta.

La obra de Huerta de Soto comienza indicando que no es posible "entender el concepto de socialismo sin comprender previamente la esencia de la función empresarial” (2015, p. 41). El concepto de acción humana está ligado a una práctica emprendedora, "por un lado, como característica esencial y eminentemente creativa de todo ser humano y, por otro lado, como el conjunto de facultades coordinadoras que hacen posible el surgimiento y desarrollo de la civilización" (íbid.). Así, la función empresarial es inherente a todas las acciones humanas, pues pretende modificar el presente para lograr los fines del actor en un futuro más o menos próximo ${ }^{5}$. Todas las acciones humanas ejercen la función empresarial; no es un atributo de un grupo o clase social. Como Mises explicó, "en cualquier economía real y viva, todo actor es siempre un emprendedor" (1949, p. 253). Dadas las preferencias y percepciones tecnológicas, los individuos actúan sobre ellas para llegar a un estado más satisfactorio, a una ganancia subjetiva. El estado preferido que el actor espera lograr es su 'fin', los pasos por los cuales el actor intenta conseguir su meta son los 'medios'.

Huerta de Soto explica que los vínculos interpersonales dan origen a la sociedad como "un proceso dinámico de interacciones humanas movidas por la fuerza de la función empresarial, que hacen posible la división del conocimiento y la vida en común con una complejidad y riqueza de matices y elementos cada vez mayores" (2015, p. 85). Si una sociedad se basa en vínculos contractuales, los individuos tienen derechos de propiedad privada: son dueños de su persona y fruto de su creatividad empresarial y de su libre uso (Rothbard, 1973; Hoppe, 2012). Si bien la sociedad es una agrupación pactada de personas, organizada para cooperar en la consecución de determinados fines, el mercado es un conjunto de intercambios de derechos de propiedad

5 Emprendimiento viene etimológicamente del verbo latino in prehendoendi-ensum, que significa "descubrir, percibir, identificar, realizar". La Real Academia Española (2021) define empresa como una "acción que involucra dificultades y cuya ejecución requiere decisión y esfuerzo”, y como el “intento o diseño de hacer algo", es decir, una acción. Un empresario es aquel que "se compromete con acciones de resolución", algo "propio de las personas". En la ciencia económica se acepta ampliamente que la función empresarial, como habilidad inherente a todos los seres humanos, es la esencia de los fenómenos económicos (Shane y Venkataraman, 2000; Parker, 2018; Ravier, 2020). Las cursivas son propias. 
realizadas libremente por los agentes económicos (RAE, 2021). Por ello, Huerta de Soto dice que sociedad y mercado son dos caras de la misma moneda.

La comprensión del proceso del mercado lleva al principio de que el cálculo económico -la estimación en unidades monetarias de los resultados de diferentes planes de acción- solo es posible si existe un sistema de precios, relaciones históricas de intercambio expresadas en dinero. Los precios de mercado surgen cuando hay intercambios voluntarios impulsados por valoraciones subjetivas de los individuos. Cada consumidor decide bienes y servicios en el mercado, mientras que cada empresario valora, estima y pronostica precios futuros que sus consumidores potenciales pueden aceptar. Como muestra Huerta de Soto, un entorno institucional favorable a la propiedad privada y a los vínculos contractuales tiende a "fortalecer el proceso empresarial de creación y transmisión de información y coordinación social" (2015, p. 74). El proceso del mercado es eficiente entonces porque los individuos solo prosperan si ajustan continuamente su intelecto para satisfacer los requisitos urgentes de los demás.

Por tanto, el desarrollo económico como "la ampliación del rango de alternativas abiertas a la gente" requiere instituciones económicas "inclusivas", que crean incentivos y oportunidades de amplia base en la sociedad (Espinosa, 2020). Las instituciones, tales como el lenguaje, la propiedad privada, el derecho, el dinero, despliega el potencial creativo y coordinador de la función empresarial para producir más y mejores soluciones a los problemas humanos, además de reducir los costos de transacción y la incertidumbre (Bjørnskov y Foss, 2016; Bylund y McCaffrey, 2017; Acemoglu y Robinson, 2019). En cambio, las instituciones "extractivas", que carecen de estas propiedades, crean pobreza. Los entornos hostiles a la propiedad privada y a los vínculos contractuales "debilitan el potencial creativo y coordinador de la función empresarial” (Huerta de Soto, 2015, p. 75).

En consecuencia, la teoría económica de Huerta de Soto, basada en el individualismo metodológico, no es un razonamiento circular. Es una construcción de "cadenas verbales de la lógica", desde la esencia -la acción humana del individuo dotado de una habilidad creativa y empresarial innata- hasta el análisis institucional: una teoría de los intercambios. Por ello, la acusación de Nieto sobre el razonamiento circular solo tiene sentido en un marco dialéctico y materialista que desatiende el análisis lógico y deductivo del conocimiento teórico $a$ priori: la categoría de la acción humana como el elemento pivote de toda la ciencia económica. Por lo demás, las teorías de Mises, Hayek 
y Huerta de Soto no son enfoques distintos de investigación de la economía, más bien son como una theoretical matryoshka que ajusta, afina y amplía el núcleo del análisis (Boettke, 2019).

Por último, el enfoque de Nieto de que la función empresarial y la búsqueda del beneficio "no es un rasgo de la naturaleza humana" implica una auto-contradicción: ¡él actúa de forma emprendedora! Busca medios -la teoría marxista- para lograr su fin que valora más subjetivamente: refutar la teoría austriaca de la imposibilidad del socialismo. Si piensa que su refutación es conveniente para superar el desafío de los austriacos, obtendrá una ganancia subjetiva (el valor del fin logrado supera a los costes). De lo contrario, tendrá una pérdida subjetiva (el valor del fin logrado es inferior a los costes). ¿Significa esto que Nieto no comparte los rasgos de la naturaleza humana? Es justo al revés.

\section{LA IMPOSIBILIDAD DEL CIBER-COMUNISMO}

La tesis principal de Nieto es que "el argumento austriaco [...] no impugna la posibilidad de [...] un modelo de economía planificada que tenga en cuenta las posibilidades tecnológicas actuales" (2020, 139). El desafío de los austriacos quedaría sin efecto con el cibercomunismo: la planificación informatizada sin derechos de propiedad privada mediante las tecnologías de la información y la comunicación (TIC) (Cockshott, 2017). Así, Nieto afirma que el marco institucional ciber-comunista es compatible con la eficiencia dinámica, es decir, la economización de los recursos y la coordinación social de los planes individuales ${ }^{6}$. La planificación de la economía mediante la tecnología actual sería más conveniente para promover el desarrollo genuino de las preferencias y necesidades reales de la población.

E1 ciber-comunismo se basa en cuatro desarrollos de las fuerzas productivas desde 1960:1) los supercomputadores pueden resolver millones de ecuaciones en segundos (la objeción de Mises); 2) el internet hace posible la planificación cibernética en tiempo real y resuelve los problemas del conocimiento disperso (la objeción de Hayek); 3) los big data permiten concentrar la información necesaria

\footnotetext{
${ }^{6}$ Marx y Engels sugieren que el comunismo se puede "resumir en una frase: abolición de la propiedad privada” (1848, p. 498). El comunismo está relacionado con la expropiación de los medios de producción para lograr una "verdadera armonía de intereses". En el comunismo, dice Nieto, "cada uno determina sus preferencias y su plan de vida, mientras que las decisiones que toma el colectivo se toman de forma democrática, no por imposición de un propietario minoritario" (2020, p. 148).
} 
para planificar; 4) las tarjetas de pago electrónico permiten sustituir el efectivo por créditos laborales intransferibles.

Cockshott (2017) sugiere que "la complejidad computacional para determinar el valor trabajo de una economía con $N$ productos distintos crece como $N \log (N)$ ". Así, la información de las acciones humanas es "tratable y fácilmente resuelta por los computadores más modernas" (p. 29). Además, Cottrell y Cockshott $(1993,2008)$ indican que el cálculo en tiempo de trabajo -valor promedio creado por hora- es un proceso racional cuando los algoritmos guían eficientemente la asignación de recursos, lo que serviría para eliminar la incertidumbre empresarial. Igualmente, Cockshott y Cottrell (2000) y Cockshott y Nieto (2017) describen los fundamentos del ciber-comunismo sobre dos pilares. Primero, la democracia directa implica que las mayores decisiones estratégicas se toman democráticamente. ¿Cuánto trabajo asignar a educación, salud, pensiones, protección del medio ambiente, defensa nacional, inversión? Todo esto y más se podría lograr por votación directa usando computadores o teléfonos móviles. Segundo, la economía equitativa señala que los bienes se distribuyen según el principio de equivalencia: cada persona obtiene en bienes la misma cantidad de trabajo que realiza, después de impuestos. Los bienes se cotizan en horas laborales, donde la retroalimentación cibernética de las ventas coordina la producción a las necesidades de las personas. De ahí la necesidad de desarrollar un sistema de Internet centralizado para rastrear todas las compras y ventas. En definitiva, estos autores defienden que la transición al cálculo ciber-comunista conducirá hacia el uso racional del tiempo de trabajo y, por lo tanto, un crecimiento más rápido de la productividad laboral.

Sin embargo, hay dos razones principales que explican por qué la promesa del ciber-comunismo acerca de suscitar el desarrollo genuino de la población es imposible. Por un lado, el ideal ciber-comunista es una imposibilidad lógica. Si el ciber-comunismo es la universalidad de la copropiedad entre la población, entonces ninguna persona podría decir o hacer nada sin la aprobación del resto de la comunidad. ¿Cómo podría alguien dar tal consentimiento sin ser el propietario exclusivo de su propio cuerpo y creatividad empresarial? ¿Quién decide qué decisiones del distrito individual al colectivo? Es inevitable concluir que el ciber-comunismo es una utopía. Por eso, el socialismo, la fase previa, resulta en una oligarquía de expropiadores que se erigen como dueños del cuerpo, la creación y el trabajo de los gobernados. Esta es la realidad del comunismo: el fortalecimiento de regímenes socialistas totalitarios (Ebeling, 2015; Boettke, 2020). 
Al presentar un mundo de ficción como criterio de comparación, los ciber-comunistas crean una falsa impresión de conocimiento científico. Sin propiedad privada, no puede haber función empresarial productiva (basada en vínculos contractuales y precios de mercado) porque los individuos están sometidos a los designios del colectivo. La ausencia de propiedad privada hace que la democracia directa sea un absurdo. Si el individuo no existe y solo importa lo colectivo, ¿por qué pedir la opinión de los individuos? ¿Cómo decidirán si no son dueños de su cuerpo y de la creatividad empresarial? La imposibilidad de la copropiedad comunal hace inevitable la aparición de un nuevo órgano director: una oligarquía explotadora (Holcombe, 2004). Sin propiedad privada, i.e., sin cálculo económico, ¿quién decidirá cuánto trabajo se debe asignar a cada una de las actividades económicas? Un órgano de gobierno decidirá sobre todos los asuntos que conciernen al colectivo, es decir, la vida de todos los individuos. Si las decisiones económicas y empresariales se determinan con criterios políticos, será imposible considerar criterios de rentabilidad si por casualidad hubiese sido posible conseguir el mismo objetivo con menos mano de obra y medios materiales (objeción de Mises) ${ }^{7}$. Independientemente de una gobernanza basada en datos, en ausencia de propiedad privada, el cálculo económico es imposible y la niebla de ignorancia de los tomadores de decisiones ciber-comunistas seguramente será mayor (Boettke y Leeson, 2005; Kirzner, 2017).

Por otro lado, la abolición de los derechos de propiedad privada es un ataque a la esencia del desarrollo económico: la capacidad creativa y empresarial de los individuos para identificar y resolver problemas humanos (Espinosa et al., 2020). Por eso, la naturaleza fundamental del ciber-comunismo es la violencia para imponer ciertos objetivos y visiones del mundo. En este sentido, la idea de la lucha de clases solo tiene sentido si es inducido por determinados grupos de individuos; no es inherente a una clase como señalan los marxistas. Además, si los intercambios son voluntarios (respeto a la propiedad privada de los demás), no puede haber explotación (por definición, los intercambios voluntarios son mutuamente beneficiosos a priori). Como dijo Huerta

7 La entrega de cupones en horas de trabajo tiene dos problemas insolubles, incluso con la ayuda de las nuevas tecnologías: 1) el criterio de las horas de trabajo no aplica a la creatividad empresarial -ya que no exige medios materiales para ser ejercida- y tampoco a los recursos naturales no reproducibles -ya que permiten lograr fines sin utilizar horas de trabajo-; 2) el factor trabajo no existe, sino innumerables categorías y clases distintas de trabajo (Böhm-Bawerk, 1896, 1959, 2, pp. 248-256; Mayer, 1994; Boettke y Candela, 2017).

Revista de Economía Institucional, vol. 23 , N. ${ }^{\circ} 45$, Segundo Semestre/202i, pp. i63-i83 ISSN OI $24-5996 / \mathrm{E}-$ ISSN 2346-2450 
de Soto, la praxis del comunismo se entiende mejor como un "sistema de agresión institucional contra el ejercicio de la función empresarial" (2015, p. 49). Sin embargo, ¿el ciber-comunismo puede promover la eficiencia dinámica, al menos como la economía de mercado?

Otra vez, las decisiones ciber-comunistas definidas ex ante por el colectivo se limitarán a coordinar las acciones de los individuos con respecto a las necesidades del órgano de gobierno, ya sea unipersonal o colegiado ${ }^{8}$. Si las personas no pueden ejercer libremente su función empresarial, es imposible lograr una verdadera armonía de intereses (objeción de Hayek). Las personas coaccionadas perciben que pueden tener más posibilidades de lograr sus objetivos si usan su creatividad para influir en las decisiones políticas: es el efecto de la corrupción, es decir, la función empresarial improductiva o destructiva. Igualmente, mayores riesgos de confiscación a la propiedad privada (p. ej., altos niveles de impuestos, inflación, barreras comerciales, corrupción del sector público, riesgo de default y expropiación) anticipan menores niveles de función empresarial productiva (Rothbard, 1970; Baumol, 1990, Ikeda, 2015). Como revela Huerta de Soto, “en ausencia de los procesos de mercado, los juicios comparativos sobre distintos tipos de trabajo habrán de ser arbitrarios" (2015, p. 206). En consecuencia, el ciber-comunismo será dinámicamente ineficiente porque provoca un atraso económico, tecnológico y cultural ${ }^{9}$.

Aunque el cálculo económico no siempre garantiza la coordinación de los planes individuales, las soluciones perfectas están más allá de las posibilidades humanas. En todo caso, los procesos de mercado sin barreras institucionales tienden a fomentar el desarrollo económico a través de la competencia para confiar dinámicamente la dirección de la producción a las personas más astutas para satisfacer las demandas de los demás (Foss y Klein, 2017; Cachanosky, 2020). Es un proceso incesante como la propagación de un big bang social coordinador.

${ }^{8}$ Espinosa (2021) analiza la decepción con el proyecto Cybersyn en Chile, creado por Stafford Beer, para implementar la planificación cibernética en tiempo real durante el gobierno de Salvador Allende (1970-1973).

9 Como ejercicio teórico, el ideal comunista podría lograrse mejor con un anarquismo de propiedad privada o anarcocapitalismo, donde la función empresarial promueva el progreso tecnológico y su eficiencia dinámica. Está más allá del alcance de este artículo rastrear los principios institucionales del anarcocapitalismo, y remitimos a Rothbard, (1973), Huerta de Soto (2007), Block (2011) y Friedman (2014). 


\section{CONCLUSIONES}

Aunque se han atenuado los lapsos más crudos del marxismo, todavía están muy extendidos. Su exposición inmediata se ha vuelto más difícil en parte por la gran confusión entre el avance del conocimiento y la promoción de la política, y la subordinación del primero a la segunda en detrimento de ambos; y en parte por la construcción de una fachada de recursos tecnológicos - big data y supercomputadores, técnicas matemáticas y métodos econométricos- que a veces actúan como pantalla protectora para justificar una utopía. El artículo de Nieto sobre la posibilidad del cálculo socialista basado en la institucionalidad ciber-comunista no ofrece una solución consistente al desafío de los austriacos. Algunas de sus opiniones son sostenibles, otras son difíciles de justificar, pero la mayoría de los argumentos en que se apoya son inexactos. Por ello, exponer las principales deficiencias del ciber-comunismo requiere algo más que el simple dominio del análisis económico: requiere observación, reflexión y cierto conocimiento de la historia. Ante tales deficiencias y errores, el desafío de los austriacos sigue siendo sólido.

\section{REFERENCIAS BIBLIOGRÁFICAS}

Acemoglu, D. y Robinson, J. A. (2019). Rents and economic development: The perspective of Why nations fail. Public Choice, 181(1-2), 13-28.

Arnaert, B. L. (2018). Talking to walls: the socialist calculation debate that never really was one. Procesos de Mercado, 15(2), 339-357.

Baumol, W. J. 1990. Entrepreneurship: productive, unproductive, and destructive. Journal of Political Economy, 98(5), 893-921.

Bjørnskov, C. y Foss, N. J. (2016). Institutions, entrepreneurship, and economic growth: what do we know and what do we still need to know? Academy of Management Perspectives, 3O(3), 292-315.

Block, W. E. (2011). Governmental inevitability: reply to Holcombe. Journal of Libertarian Studies, 22, 667-688.

Böhm-Bawerk, E. V. [1896] (1949). Karl Marx and the close of his system. Nueva York: Augustus M. Kelley.

Böhm-Bawerk, E. V. (1959). Capital and interest, v. 2, Positive theory of capital. South Holland: The Libertarian Press.

Boettke, P. J. (2000). Socialism and the market: the socialist calculation debate re-visited. Londres y Nueva York: Routledge.

Boettke, P. J. (2019). How Misesian was the Hayekian research program? Procesos de Mercado, 16(1), 251-257.

Boettke, P. J. (2020). Property, predation and socialist reality. Journal of Institutional Economics, 16(2), 185-197.

Boettke, P. J. y Candela, R. (2017). Price theory as prophylactic against popular fallacies. Journal of Institutional Economics, 13(3), 725-752. 
Boettke, P. J. y Leeson, P. T. (2005). Still impossible after all these years: reply to Caplan. Critical Review, 17(1-2), 155-170.

Bylund, P. L. y McCaffrey, M. (2017). A theory of entrepreneurship and institutional uncertainty. Journal of Business Venturing, 32(5), 461-475.

Cachanosky, I. (2020). En defensa del monopolio competitivo. Procesos de Mercado, 17(1), 233-264.

Cachanosky, J. (1994). Historia de las teorías del valor y del precio, parte I. Revista Libertas, 20(1), 1-100.

Cachanosky, J. (1995). Historia de las teorías del valor y del precio, parte II. Revista Libertas, 22(1), 1-42.

Caldwell, B. (1997). Hayek and socialism. Journal of Economic Literature, 35(4), 1856-1890.

Caldwell, B. (2008). Hayek's challenge: An intellectual biography of FA Hayek. Chicago: Chicago University Press.

Cockshott, P. (2017). Big data and super-computers: Foundations of cyber communism. Ninth International Vanguard Scientific Conference on "100 Years of real socialism and the theory of post-capitalist civilization", Hanoi, [http://eprints.gla.ac.uk/148529/].

Cockshott, P. y Cottrell A. (2000). Towards a new Socialism. Nottingham: Spokeman.

Cockshott, P. y Nieto, M. (2017). Ciber-comunismo. Planificación económica, computadoras y democracia. Madrid: Trotta.

Cottrell, A. y Cockshott, P. (1993). Calculation, complexity and planning: The socialist calculation debate once again. Review of Political Economy, 5(1), 73-112.

Cottrell, A. y Cockshott, P. (2008). Computadores y democracia económica. Revista de Economía Institucional, 10(19), 161-205.

Ebeling, R. M. (2015). F. A. Hayek and why government can't manage society. Procesos de Mercado, 12(2), 343-359.

Espinosa, V. I. (2017). Ludwig von Mises y el rol del economista: un enfoque histórico. Estudios Públicos, 146(1), 185-211.

Espinosa, V. I. (2020). Epistemological problems of development economics. Procesos de Mercado, 17(1), 55-93.

Espinosa, V. I. (2021). Salvador Allende's development policy: Lessons after 50 years. Economic Affairs, 41(1), 96-110.

Espinosa, V., Wang, W. y Hai-J., Z. (2020). Israel Kirzner on dynamic efficiency and economic development. Procesos de Mercado, 17(2), 271-298.

Evans, A. J. (2010). Only individuals choose. En P. J. Boettke (comp.), Handbook on contemporary Austrian economics (pp. 3-13). Northampton y Cheltenham: Edward Elgar.

Foss, N. J. y Klein, P. G. (2017). Entrepreneurial discovery or creation? In search of the middle ground. Academy of Management Review, 42(4), 733-736.

Friedman, D. (2014). The machinery of freedom. Chicago: Open Court.

Gray, A. (1946). The socialist tradition: Moses to Lenin. Londres y Nueva York: Longmans, Green.

Hayek, F. A. (1935). Collectivist economic planning. Londres: Routledge \& Kegan Paul. 
Hayek, F. A. (1944). The road to serfdom. Londres y Nueva York: Routledge. Hayek, F. A. (1945). The use of knowledge in society. American Economic Review, 35(4), 519-530.

Hayek, F. A. (1988). The fatal conceit: The errors of socialism. En W. W. Bartley III (comp.), The collected works of Friedrich August Hayek, v. I. Londres y Nueva York: Routledge.

Holcombe, R. G. (2004). Government: unnecessary but inevitable. Independent Review, 8(3), 325-342.

Hoppe, H. H. (1995). Economic science and the Austrian method. Auburn: The Mises Institute Press.

Hoppe, H. H. (2012). La ética y la economía de la propiedad privada. Auburn: The Mises Institute Press.

Huerta de Soto, J. (2004a). Método y crisis en la ciencia económica. En J. Huerta de Soto (comp.), Estudios de economía politica (pp. 59-82). Madrid: Unión Editorial.

Huerta de Soto, J. (2004b). Historia, ciencia económica y ética social. En J. Huerta de Soto (comp.), Estudios de economía politica (pp. 105109). Madrid: Unión Editorial.

Huerta de Soto, J. (2007). Liberalismo versus anarcocapitalismo. Procesos de Mercado, 4(2), 13-32.

Huerta de Soto, J. (2015). Socialismo, cálculo económico y función empresarial. Madrid: Unión Editorial.

Ikeda, S. (2015). Dynamics of interventionism. En P. J. Boettke y C. J. Coyne (comps.), Oxford handbook of Austrian economics (pp. 393-416). Nueva York: Oxford University Press.

Kirzner, I. M. (2017). The entrepreneurial market process an exposition. Southern Economic Journal, 83(4), 855-868.

Lavoie, D. (1985). Rivalry and central planning: the socialist calculation debate reconsidered. Nueva York: Cambridge University Press.

Marx, K. [1843] (1970). Critique of Hegel's' philosophy of right. Nueva York: Cambridge University Press.

Marx, K. [1847] (1986). La misère de la philosophie. París: LibrairesÉditeurs.

Marx, K. [1867] (1976). Capital: critique of political economy. Nueva York: Penguin Press.

Marx, K. [1885] (1978). Capital, v. 2. Nueva York: Penguin Press.

Marx, K. [1894] (1981). Capital, v. 3. Nueva York: Penguin Press.

Marx, K. y Engels, F. [1848] (1976). Manifesto of the Communist Party. En Marx-Engels collected works, v. 6 (pp. 477-519). Nueva York: International Publishers.

Marx, K. y Engels, F. [1932] (1976). The German ideology. En MarxEngels collected works, v. 5 (pp. 19-539). Nueva York: International Publishers.

Mayer, H. (1994). The cognitive value of functional theories of price. En I. Kirzner (comp.), Classics in Austrian Economics, v. II (pp. 55-168). Londres: William Pickering.

Menger, C. [1871] (1976). Principles of economics. Nueva York: Nueva York University Press. 
Mises, L. [1920] (1935). Economic calculation in the socialist commonwealth. En F. A. Hayek (comp.), Collectivist economic planning (pp. 87-130). Londres: Routledge \& Kegan Paul.

Mises, L. [1922] (1951). Socialism: an economic and sociological analysis. New Haven: Yale University Press.

Mises, L. [1949] (1966). Human action: a treatise on economics. Chicago: Henry Regnery.

Mises, L. (1957). Theory and history. New Haven: Yale University Press.

Mises, L. (2006). Marxism unmasked: From delusion to destruction. Nueva York: Foundation for Economic Education.

Nieto F., M. (2020). ¿Es imposible el cálculo económico en el socialismo? Crítica a la nueva lectura austríaca. Revista de Economía Institucional, 22(42), 127-151.

Parker, S. C. (2018). Entrepreneurship and economic theory. Oxford Review of Economic Policy, 34(4), 540-564.

Ravier, L. (2020). A redefinition of the entrepreneurial function concept. Procesos de Mercado, 17(1), 295-311.

Real Academia Española. (2021). Diccionario de la lengua española, 23. ${ }^{a}$ ed., [https://dle.rae.es].

Reisman, G. (2018). Marxism/socialism: A contribution to its death. Laguna Hills, CA: TJS Books.

Rothbard, M. N. (1951). Praxeology: reply to Mr. Schuller. American Economic Review, 41(5), 943-946.

Rothbard, M. N. [1970] (1977). Power and market: government and the economy. Kansas City: Sheed Andrews and McMeel.

Rothbard, M. N. [1973] (2002). For a new liberty: the libertarian manifesto. Auburn: The Mises Institute Press.

Rothbard, M. N. (1995). An Austrian perspective on the bistory of economic thought. Northampton y Cheltenham: Edward Elgar.

Schutz, A. (2011). Choice and social sciences. En L. Embree (comp.), Collected papers $V$. Phenomenology and the social sciences (pp. 75-92). Nueva York: Springer.

Selgin, G. A. (1988). Praxeology and understanding: an analysis of the controversy in austrian economics. Review of Austrian Economics, 2(1), 19-58.

Shafarevich, I. R. (1980). The socialist phenomenon. Nueva York: Harper \& Row.

Shane, S. y Venkataraman, S. (2000). The promise of entrepreneurship as a field of research. Academy of Management Revierw, 25(1), 217-226.

Steele, D. R. (2013). From Marx to Mises: post capitalist society and the challenge of economic calculation. Chicago: Open Court. 